\title{
Multisystem mitochondrial diseases due to mutations in mtDNA-encoded subunits of complex I
}

Tereza Danhelovska', Hana Kolarova', Jiri Zeman', Hana Hansikova', Manuela Vaneckova², Lukas Lambert², Vendula Kucerova-Vidrova', Kamila Berankova', Tomas Honzik' and Marketa Tesarova ${ }^{1 *}$ (D)

\begin{abstract}
Background: Maternally inherited complex I deficiencies due to mutations in MT-ND genes represent a heterogeneous group of multisystem mitochondrial disorders (MD) with a unfavourable prognosis. The aim of the study was to characterize the impact of the mutations in MT-ND genes, including the novel m.13091 T > C variant, on the course of the disease, and to analyse the activities of respiratory chain complexes, the amount of protein subunits, and the mitochondrial energy-generating system (MEGS) in available muscle biopsies and cultivated fibroblasts.
\end{abstract}

Methods: The respiratory chain complex activities were measured by spectrophotometry, MEGS were analysed using radiolabelled substrates, and protein amount by SDS-PAGE or BN-PAGE in muscle or fibroblasts.

Results: In our cohort of 106 unrelated families carrying different mtDNA mutations, we found heteroplasmic mutations in the genes MT-ND1, MT-ND3, and MT-ND5, including the novel variant m.13091 T > C, in 13 patients with MD from 12 families. First symptoms developed between early childhood and adolescence and progressed to multisystem disease with a phenotype of Leigh or MELAS syndromes. MRI revealed bilateral symmetrical involvement of deep grey matter typical of Leigh syndrome in 6 children, cortical/white matter stroke-like lesions suggesting MELAS syndrome in 3 patients, and a combination of cortico-subcortical lesions and grey matter involvement in 4 patients. MEGS indicated mitochondrial disturbances in all available muscle samples, as well as a significantly decreased oxidation of $\left[1-{ }^{14} \mathrm{C}\right]$ pyruvate in fibroblasts. Spectrophotometric analyses revealed a low activity of complex I and/or complex I + III in all muscle samples except one, but the activities in fibroblasts were mostly normal. No correlation was found between complex I activities and mtDNA mutation load, but higher levels of heteroplasmy were generally found in more severely affected patients.

Conclusions: Maternally inherited complex I deficiencies were found in $11 \%$ of families with mitochondrial diseases in our region. Six patients manifested with Leigh, three with MELAS. The remaining four patients presented with an overlap between these two syndromes. MEGS, especially the oxidation of $\left[1-{ }^{14} \mathrm{C}\right]$ pyruvate in fibroblasts might serve as a sensitive indicator of functional impairment due to MT-ND mutations. Early onset of the disease and higher level of mtDNA heteroplasmy were associated with a worse prognosis.

Keywords: mtDNA, MT-ND genes, Complex I, Leigh syndrome, MELAS syndrome, MEGS, Mitochondria

\footnotetext{
* Correspondence: marketa.tesarova@lf1.cuni.cz

${ }^{1}$ Department of Pediatrics and Adolescent Medicine, First Faculty of

Medicine, Charles University and General University Hospital in Prague, Ke

Karlovu 2, 12808 Praha 2, Prague, Czech Republic

Full list of author information is available at the end of the article
}

(c) The Author(s). 2020 Open Access This article is distributed under the terms of the Creative Commons Attribution 4.0 International License (http://creativecommons.org/licenses/by/4.0/), which permits unrestricted use, distribution, and

reproduction in any medium, provided you give appropriate credit to the original author(s) and the source, provide a link to the Creative Commons license, and indicate if changes were made. The Creative Commons Public Domain Dedication waiver (http://creativecommons.org/publicdomain/zero/1.0/) applies to the data made available in this article, unless otherwise stated. 


\section{Background}

Disturbances of the respiratory chain complex I (CI, NADH:coenzyme Q oxidoreductase, EC 1.6.5.3) represent the most common cause of multisystem mitochondrial disorders (MD), accounting for nearly one-third of patients [1]. CI consists of 45 protein subunits with different functions necessary for enzyme assembly, stabilization, and regulation [2], encoded by genes in nuclear or mitochondrial DNA (mtDNA). Many mutations in these genes have already been described including in 22 genes for structural proteins and 11 genes for non-structural proteins encoded by nuclear DNA (Additional file 1: Table S1), and in all $7 \mathrm{mtDNA}$ genes (MTND1-6 and MT-ND4L) for structural subunits of CI. With respect to maternally inherited mutations, 28 different mutations sites have been confirmed, and another 113 sites in $M T-N D$ genes have been published (www.mitomap.org).

Clinically, CI deficiency represents a heterogeneous group of MDs with an early, neonatal onset of fatal lactic acidosis; infantile onset of progressive mitochondrial encephalopathy with Leigh syndrome (LS); onset of Mitochondrial Encephalopathy, Lactic Acidosis and Stroke-like episodes (MELAS) syndrome during childhood, or adult-onset encephalomyopathic syndromes with various severities. Leber Hereditary Optic Neuropathy (LHON) syndrome with acute or subacute loss of vision usually starts during the second or third decade of life. In addition, several studies have also documented the LHON/MELAS overlap syndromes [3-5].

The prognosis in patients with maternally inherited complex I deficiencies is unfavourable and hardly predictable. In the cohort of 13 patients with MD due to 8 different mtDNA mutations in $M T-N D 1, M T-N D 3$ and $M T-N D 5$ genes, including one novel variant m.13091 $\mathrm{T}>\mathrm{C}$ in $M T-N D 5$ gene, we characterized the impact of the mutations in $M T-N D$ genes on the course of the disease and we analysed their biochemical consequences in available muscle biopsies and cultivated skin fibroblasts.

\section{Material and methods \\ Patients}

Our laboratory serves as the diagnostic centre for MD in the Czech Republic, a country with 10.5 million inhabitants. During the last 25 years, different maternally inherited mtDNA mutations have been diagnosed in 106 unrelated families and sporadic large-scale deletions in mtDNA in 25 patients with Kearns-Sayre/Pearson syndromes. mtDNA mutations in genes for structural subunits of CI were present in 47 families, including 12 families with 13 patients with multisystem diseases due to heteroplasmic mtDNA mutations in $M T-N D 1, M T$ ND3 and MT-ND5, and 35 families with CI deficiency and LHON syndrome with tissue-specific optic nerve involvement due to homoplasmic mutations in $\mathrm{m} .3460 \mathrm{G}>\mathrm{A}$ in $M T-N D 1, \mathrm{~m} .11777 \mathrm{G}>\mathrm{A}$ in $M T-N D 4$ and $\mathrm{m} .14484 \mathrm{~T}>\mathrm{C}$ in $M T-N D 6$.

\section{Methods \\ Analysis of mtDNA}

In patients $1-8$ and 10, total genomic DNA isolated from muscle biopsy or cultivated skin fibroblasts (P2) and mtDNA (NC_012920) was sequenced as described previously [6]. In patients 9 and 11-13, the mtDNA mutation m.13513G $>$ A was detected by PCR-RFLP using mismatch primers (F: 5'-GTTTGCGGTTTCGA TGATGTGAT-3'; R: 5' - AACCATACC-TCTCAC TTCAACCTCCC-3') and Bsp143I endonuclease (Thermo Fisher Scientific, Waltham, Massachusetts, USA). Levels of heteroplasmy in available tissues were determined by mutation-specific PCR-RFLP analyses. Digested products were separated on an Agilent 2100 Bioanalyzer using High Sensitivity DNA kits. For each sample, the intensities of the individual restriction fragments were determined using Agilent 2100 Expert Software (Agilent Technologies, Santa Clara, California, USA). The level of heteroplasmy was calculated as the percentage of fragment intensity corresponding to the mutated mtDNA molecule. The detection limit of this method is $3 \%$.

\section{Isolation of mitochondria}

Samples obtained by muscle biopsy were transported on ice (at $4{ }^{\circ} \mathrm{C}$ ) and mitochondria were isolated immediately according to standard differential centrifugation procedures [7] in a buffer containing $150 \mathrm{mM} \mathrm{KCl}, 50 \mathrm{mM}$ Tris/ $\mathrm{HCl}, 2 \mathrm{mM}$ EDTA and $2 \mu \mathrm{g} / \mathrm{ml}$ aprotinin ( $\mathrm{pH} 7.5$ ) at $4{ }^{\circ} \mathrm{C}$. The homogenate was centrifuged for $10 \mathrm{~min}$ at $4^{\circ} \mathrm{C}$ and $600 \mathrm{~g}$, the supernatant was filtered through a $100 \mu \mathrm{m}$ nylon membrane, and mitochondria were obtained by centrifugation for $10 \mathrm{~min}$ at $4{ }^{\circ} \mathrm{C}$ and $10,000 \mathrm{~g}$. The mitochondrial pellet was washed and resuspended to a final protein concentration of $20-25 \mathrm{mg} / \mathrm{ml}$ [8].

\section{Spectrophotometry}

The activities of respiratory chain complexes (complex I - NADH:coenzyme Q oxidoreductase, CI, EC 1.6.5.3; complex I + III - NADH:cytochrome $c$ oxidoreductase, CI + III; complex II - succinate:coenzyme Q oxidoreductase, CII, EC 1.3.5.1; complex II + III - succinate:cytochrome $c$ oxidoreductase, CII + III; complex III - coenzyme Q:cytochrome $c$ oxidoreductase, CIII, EC 7.1.1.8; complex IV - cytochrome $c$ oxidase, CIV, EC 1.9.3.1) were measured according to [9]. The activity of citrate synthase (CS, EC 2.3.3.1), serving as the control enzyme to avoid assay variability, was measured according to [10]. Protein concentrations were measured by the Lowry method [11]. 


\section{Electrophoresis}

Blue Native Polyacrylamide Gel Electrophoresis (BNPAGE) separation [12] of mitochondrial membrane complexes on polyacrylamide $4-14 \%$ or $6-15 \%$ (w/v) gradient gels (MiniProtean 3 System; Bio-Rad, Hercules, California, USA), followed by immunoblot analysis was used to analyse the steady-state levels of oxidative phosphorylation system complexes [13]. Primary detection of BN-PAGE blots was performed using mouse monoclonal antibodies against the CI subunit NDUFA9 (1:2000), complex II subunit SDH70 protein (1: 6666), complex III subunit Core 2 (1:20000), complex IV subunit COX1 (1:3000) and ATP synthase subunit alpha (1:2000) (Abcam, Cambridge, UK). Sodium Dodecyl Sulfate Polyacrylamide Gel Electrophoresis (SDS-PAGE) was performed on $12 \%(\mathrm{w} / \mathrm{v})$ polyacrylamide minigels (MiniProtean $^{\circ} 3$ System) according to Schägger and von Jagow [14]. Primary antibodies against the CI subunits ND5 (1: 2000), NDUFA9 (1:4000), and NDUFB6 (1:3000); complex II subunit SDH70 (1:20000); complex III subunits Core 1 (1:2000) and Core 2 (1:40000); complex IV subunit COX2 (1:10000) (all from Abcam) and control cytosol marker $\beta$-tubulin (1:4000; Sigma, St. Louis, Missouri, USA) were used for the detection of SDS-PAGE membranes. The immunoblots were detected with peroxidase-conjugated secondary antibodies and SuperSignal West Femto Maximum Sensitivity Substrate (Thermo Fisher Scientific) using G:Box (Syngene, Cambridge, UK) and analysed by Quantity One software (Bio-Rad).

\section{MEGS analysis}

The analysis of the mitochondrial energy-generating system (MEGS) was performed in 10 incubations containing ${ }^{14} \mathrm{C}$-labelled pyruvate, malate and succinate, donors and acceptors of Acetyl-CoA and inhibitors of TCA cycle, according to Janssen [15]. Briefly, each incubation contains the buffer for MEGS $\left(30 \mathrm{mM} \mathrm{KH_{2 }} \mathrm{PO}_{4} \mathrm{pH}\right.$ 7.4; $75 \mathrm{mM} \mathrm{KCl} ; 8 \mathrm{mM}$ Tris; $1.6 \mathrm{mM}$ EDTA; $5 \mathrm{mM} \mathrm{MgCl}$; $0.2 \mathrm{mM}$ p1,p5-di (adenosine-5') pentaphosphate (myoadenylate kinase inhibitor), and where indicated, $2 \mathrm{nM}$ ADP; $1 \mathrm{mM}$ pyruvate; $1 \mathrm{mM}$ malate; $1 \mathrm{mM}$ succinate (all from Sigma); with combinations of $\left[1-{ }^{14} \mathrm{C}\right]$ pyruvate (PerkinElmer, Waltham, Massachusetts, USA); [U- $\left.{ }^{14} \mathrm{C}\right]$ malate (PerkinElmer) and $\left[1,4-{ }^{14} \mathrm{C}\right]$ succinate (Moravek Biochemicals, Brea, California, USA); $5 \mathrm{mM} \mathrm{L-carnitine;}$ $2 \mathrm{mM}$ acetyl-D,L-carnitine; $2 \mathrm{mM}$ sodium arsenite; 5 $\mathrm{mM}$ malonate; $2 \mu \mathrm{M} \mathrm{CCCP}$ and $40 \mu \mathrm{M}$ atractyloside (all from Sigma). The composition of individual incubations is summarized in Additional file 1: Table S2. Incubations were performed in a shaking water bath at $37^{\circ} \mathrm{C}$ in 0.2 $\mathrm{ml}$ glass incubation vials with caps and rubber septa. The measurement was started by adding $5 \mu \mathrm{l}$ of whole cell lysate of fibroblasts and stopped after $20 \mathrm{~min}$ by
$50 \mu \mathrm{l} 3 \mathrm{M} \mathrm{HClO}_{4}$. The $\mathrm{CO}_{2}$ produced was trapped on a filter paper (saturated by $1 \mathrm{M} \mathrm{NaOH}$ ) in each cap for $1 \mathrm{~h}$ at $4{ }^{\circ} \mathrm{C}$; the filter paper was then transferred to a scintillation vial with $3 \mathrm{ml}$ BCS solution (Amersham, Little Chalfont, UK) and ${ }^{14} \mathrm{CO}_{2}$ was counted after $24 \mathrm{~h}$ in the Beckman Coulter LS6500 (Beckman Coulter, Brea, California, USA). Due to repeated measurements, the data related to protein amounts were analysed using a logistic regression model with mixed effects, where the studied reaction was perceived as a disease predictor and patient subjects or controls as a random effect. $P$-values less than 0.05 were considered statistically significant. Analysis was performed in R 3.5.1 statistical package, R Core Team (2018).

\section{Results}

Multisystem MDs caused by mtDNA mutations in genes for structural subunits of CI were diagnosed in 13 patients from 12 families (P1 and P2 were cousins; their mothers are sisters), representing $11 \%$ of families with reported mtDNA mutations in our geographical region. Altogether, 8 different heteroplasmic mtDNA mutations in the genes MT-ND1, MT-ND3, and MT-ND5 were found, including one novel variant m.13091 T $>C$ (p.Met252Thr) in MT-ND5. The mutations and the levels of mtDNA heteroplasmy in muscle biopsies, cultivated fibroblasts, and other tissues are shown in Table 1. The most frequent were mutations in MT-ND5 (69\%). The same mtDNA mutations as in patients were also found in six mothers (of P1, P4, P5, P8, P10, and P11) and in two sisters (of P4 and P10) at least in one out of three or four examined tissues (blood, urinary sediment, buccal smear or hair follicles). All were asymptomatic, except the mother of P8 who had repeated attacks of migraine. No mutations were detected in the other six mothers; the mother of P2 was not analysed.

\section{Clinical data}

Clinical data are outlined in Table 1. The onset of the disease varied from the neonatal period to 17 years, and three main phenotypes were observed: 1) Six children developed LS (P1, P2, P4, P6, P9, and P13) associated with a worse prognosis, because four of them died between the age of 16 months and 7 years. Two surviving children with LS (P6 and P13) are 6.5-year-old girls. 2) Five children developed MELAS syndrome (P3, P5, P8, $\mathrm{P} 11$, and P12) with a history of stroke-like episodes, and 3) the last two children (P7 and P10) had LHON-like onset of the disease with optic neuropathy at the age of 12 years and 6 years, respectively. Nevertheless, both of them later developed multisystem symptoms and transitioned to MELAS syndrome or LHON/MELAS overlap syndrome. In addition, P9 and P11 also had MELAS syndrome with some degree of optic neuropathy with 


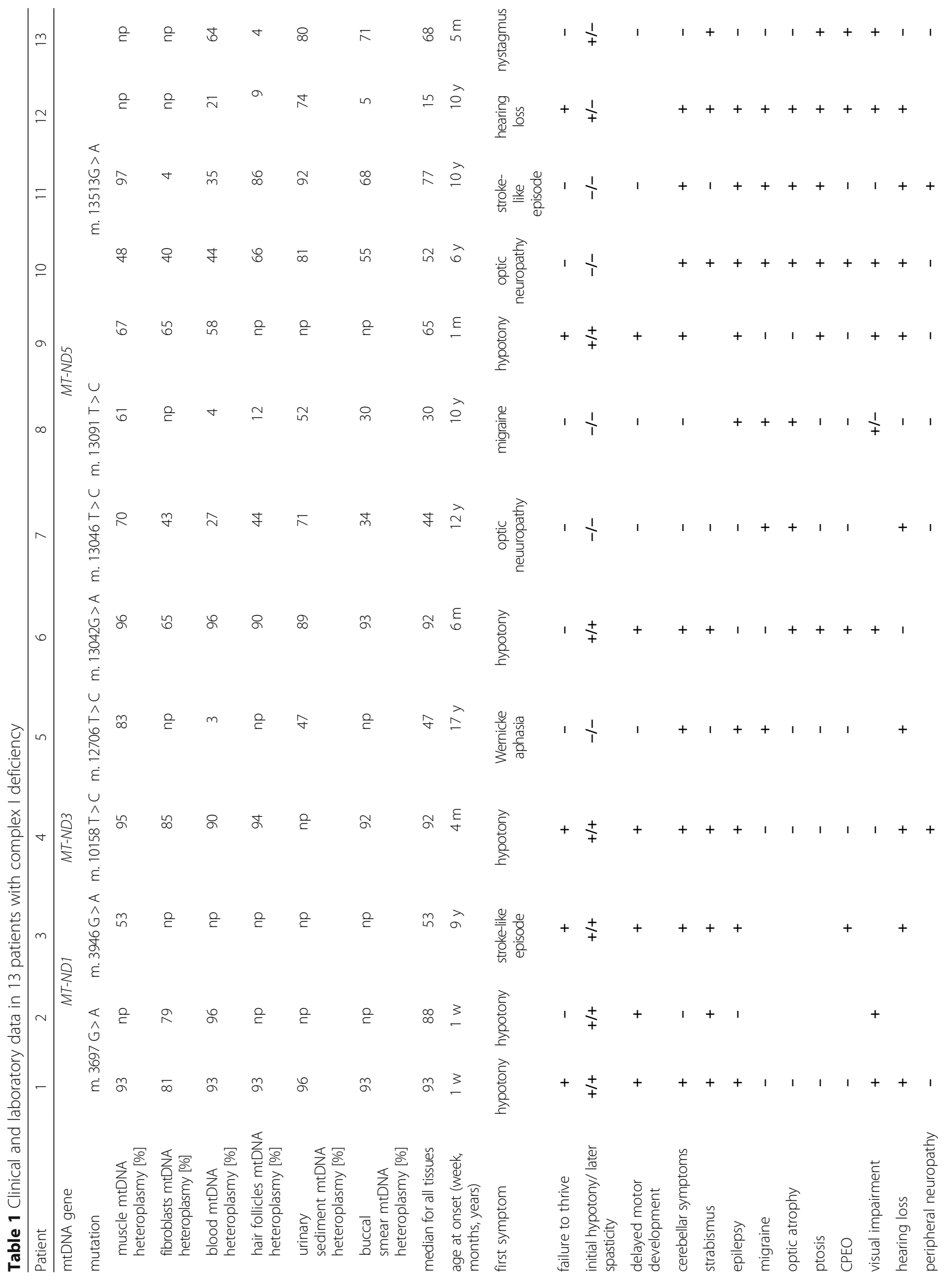


Danhelovska et al. BMC Pediatrics

(2020) 20:41

Page 5 of 13

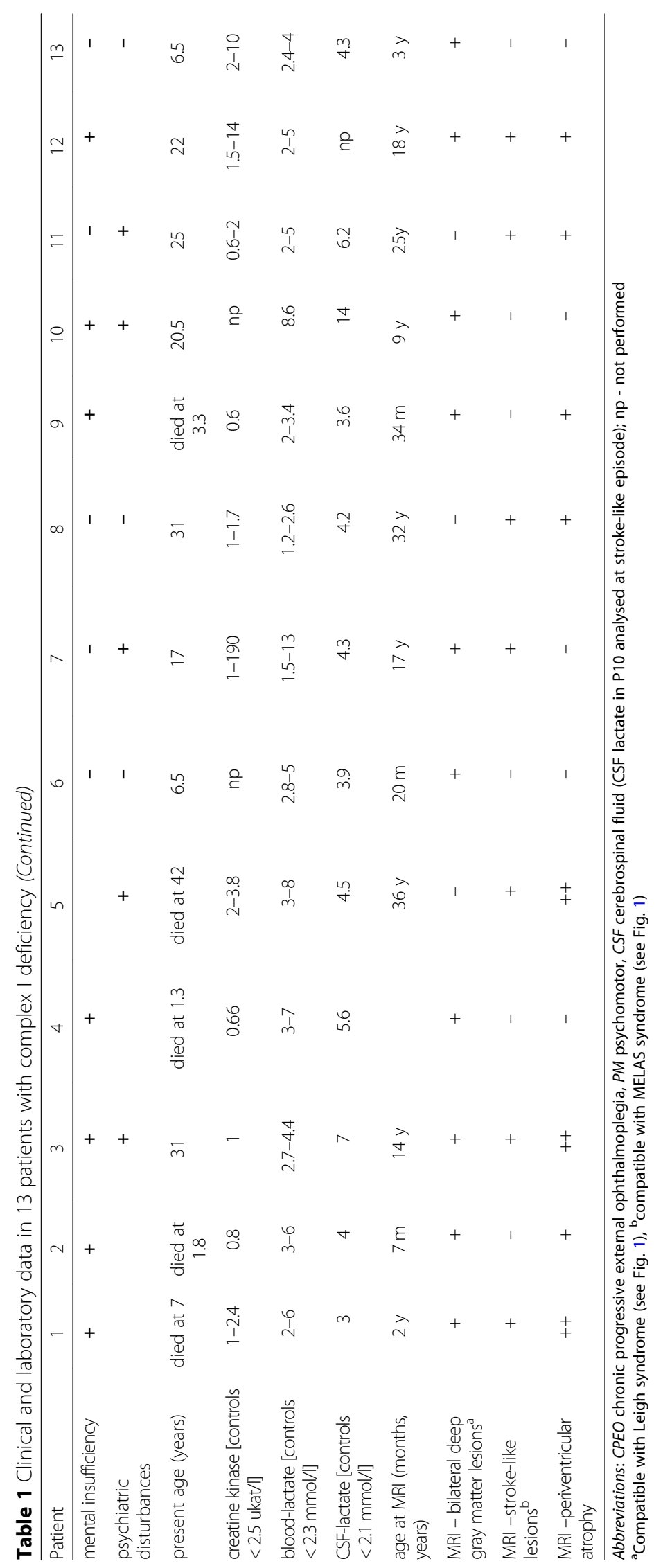


alterations in Best Corrected Visual Acuity (BCVA), and P12 had limited size of the optic discs. Hypertrophic cardiomyopathy (HCMP) with Wolf-Parkinson-White (WPW) syndrome was present in P9 and P13. Most patients also exhibited intermittent or permanent increases of lactate in the blood (B-lactate $1.2-13 \mathrm{mmol} / \mathrm{l}$, controls $<2.3 \mathrm{mmol} / \mathrm{l}$ ) and the cerebrospinal fluid (CSF-lactate 3-14 mmol/l, controls $<2.3 \mathrm{mmol} / \mathrm{l})$.

Brain MRI was performed in all patients, albeit at different ages (Table 1). In 6 of them (P2, P4, P6, P9, P10, and P13), symmetric signal changes in deep grey matter structures, including the basal ganglia and the brainstem, characteristic for LS, were found. Signal changes in the cortex and white matter of the hemispheres and the cerebellum resembling stroke-like lesions of MELAS syndrome were found in 3 patients (P5, P8, and P11), and the combination of both changes typical for LS and stroke-like lesions was present in 4 patients (P1, P3, P7, and P12). In addition, moderate to severe periventricular atrophy was found in three patients (P1, P3, and P5). Brain MRI images of all patients, except $\mathrm{P} 4$, are shown in Fig. 1.

\section{Biochemical measurement}

In isolated muscle mitochondria, the activity of $\mathrm{CI} / \mathrm{CS}$ was decreased or borderline low in 8 of 10 analysed
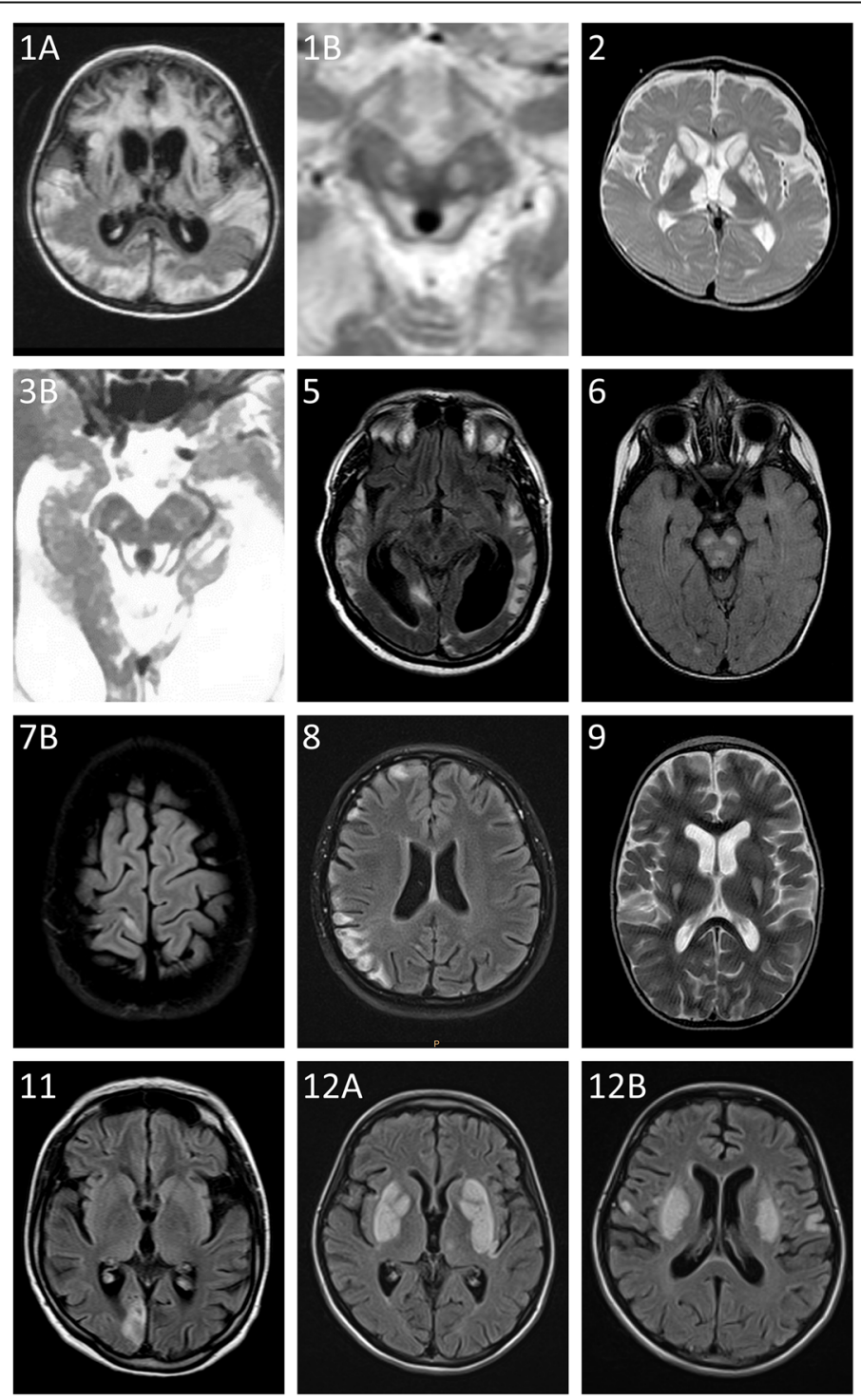
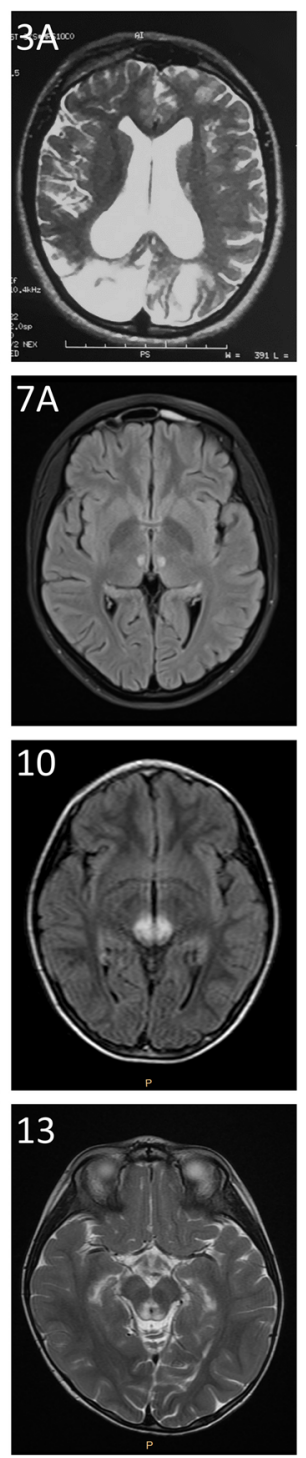

Fig. $1 \mathrm{MRI}$ of the brain in P1-P3 and P5-P12 with complex I deficiency. Signal changes in basal ganglia or brainstem characteristic for Leigh syndrome are present in patients P2, P6, P9, P10 and P13. Signal changes in the cortex and white matter of the hemispheres or cerebellum with stroke-like lesion are present in patients P5, P8, P11, and the combination of both changes are visible in patients P1, P3, P7 and P12. Moderate to severe periventricular atrophy was found in patients P1, P3 and P5. 
patients (P3-P8, P10, and P11), and the activity of the respiratory chain $\mathrm{CI}+\mathrm{III} / \mathrm{CS}$ was low in 9 of 10 analysed patients (P3-P11). Activities of other oxidative phosphorylation (OXPHOS) complexes were altered in some patients. CII + III/CS was increased in 3 patients, CIII/ CS was elevated in 3 patients, and CIV/CS was altered in 4 patients ( 3 increased, 1 decreased), see Table 2 . We observed no significant correlation between enzymatic activities and the heteroplasmy of mtDNA mutations. The activities of $\mathrm{CI}$ in cultivated fibroblasts were within the reference range (data not shown).

SDS-PAGE/Western blots (WB) in cultivated fibroblasts of patients with MT-ND5 mutations revealed slightly increased amounts of the ND5 subunit (Fig. 2a and c) in three patients (P5, P7 and P9). We noted mild alterations in the amount of selected OXPHOS protein subunits in comparison to age-related controls, but they were not uniform across the group of patients.

The MEGS analysis in cultivated skin fibroblasts from 8 patients (P1, P2, P4, P6, P7, and P9-P11) revealed decreased oxidation rates in 4 incubations containing $\left[1-{ }^{14} \mathrm{C}\right]$ pyruvate (Fig. 3). Oxidation rates of the other 2 incubations containing $\left[1-{ }^{14} \mathrm{C}\right]$ pyruvate and incubations containing $\left[\mathrm{U}_{-}{ }^{14} \mathrm{C}\right]$ malate and $\left[1,4-{ }^{14} \mathrm{C}\right]$ succinate were similar to controls. MEGS analyses were also performed in muscle postnuclear homogenate in 4 patients (P4, P5, P7 and P9). In all samples, MEGS revealed disruption at the level of the respiratory chain (data not shown).

\section{The novel variant}

The novel variant m.13091 T >C (p.Met252Thr) in the MT-ND5 gene was found in P8 (Table 1). She was born at term to healthy parents and was asymptomatic until the age of 10 years, when frequent attacks of migraine started. At the age of 26 years, she developed repeated stroke-like episodes with secondary epilepsy. She has had no ptosis or chronic ophthalmoplegia. Cardiologic examination and audiometry were uneventful, but the CSF-lactate was elevated $(4.2 \mathrm{mmol} / \mathrm{l}$, controls $<2.3 \mathrm{mmol} / \mathrm{l})$ and both optic nerves appeared pale on ophthalmoscopy. Perimetric investigation revealed multiple bilateral scotomas, and visual evoked potentials showed reduced amplitudes with prolonged P100 wave latencies. Ophthalmological assessment revealed bilateral pallor of the optic discs and decreased retinal nerve fibre layer thickness in all four quadrants (OCT Spectralis, Heidelberg Engineering, Germany). Nevertheless, the patient's visual acuity was normal, bilaterally (Best Corrected Visual Acuity 1.0). Muscle biopsy revealed a mild focal subsarcolemmal accumulation of SDH product (3\%) with a decreased activity of $\mathrm{CI}+\mathrm{III}$ in isolated mitochondria (Table 2), while the activities of $\mathrm{CI}$ and other respiratory chain complexes were within the control range, except for the increased activity of respiratory chain $\mathrm{CII}+\mathrm{III}$ (probably as the compensatory impact of CI + III deficiency). BN-PAGE revealed decreased amounts of $\mathrm{CI}$ in muscle to approx. 74\%; the amount of other OXPHOS complexes remained unchanged (Fig. 2b and d) except ATP synthase, where we observed a mildly increased amount of free $\mathrm{F}_{1}$ domain in comparison to control. Both the patient's mother and sister suffer from migraines. However, in the patient's mother, only a $12 \%$ mutational

Table 2 The activities of respiratory chain complexes in isolated muscle mitochondria

\begin{tabular}{|c|c|c|c|c|c|c|c|c|c|c|c|c|}
\hline \multirow[t]{3}{*}{ Patient } & \multicolumn{12}{|c|}{ Individual respiratory chain complexes activities in isolated muscle mitochondria } \\
\hline & \multicolumn{2}{|l|}{$\mathrm{Cl} / \mathrm{CS}$} & \multicolumn{2}{|c|}{$\mathrm{Cl}+\mathrm{III} / \mathrm{CS}$} & \multicolumn{2}{|l|}{$\mathrm{CII/CS}$} & \multicolumn{2}{|c|}{$\mathrm{Cll}+\mathrm{III/CS}$} & \multicolumn{2}{|l|}{ CIII/ CS } & \multicolumn{2}{|l|}{ CIV/CS } \\
\hline & patient & $\begin{array}{l}\text { age related } \\
\text { control } \\
\text { range }\end{array}$ & patient & $\begin{array}{l}\text { age related } \\
\text { control } \\
\text { range }\end{array}$ & patient & $\begin{array}{l}\text { age related } \\
\text { control } \\
\text { range }\end{array}$ & patient & $\begin{array}{l}\text { age related } \\
\text { control } \\
\text { range }\end{array}$ & patient & $\begin{array}{l}\text { age related } \\
\text { control } \\
\text { range }\end{array}$ & patient & $\begin{array}{l}\text { age related } \\
\text { control } \\
\text { range }\end{array}$ \\
\hline 1 & 0.50 & $0.45-1.05$ & 0.18 & $0.17-0.31$ & 0.09 & $0.07-0.27$ & 0.33 & $0.17-0.47$ & 0.43 & $0.27-0.85$ & 1.59 & $1.1-2.22$ \\
\hline 3 & 0.13 & $0.18-0.38$ & 0.08 & $0.18-0.37$ & 0.04 & $0.05-0.11$ & 0.24 & $0.17-0.32$ & 0.67 & $0.46-0.88$ & 1.21 & $0.66-2.25$ \\
\hline 4 & 0.24 & $0.45-1.05$ & 0.06 & $0.17-0.31$ & 0.09 & $0.07-0.27$ & 0.34 & $0.17-0.47$ & 0.95 & $0.27-0.85$ & 0.97 & $1.1-2.22$ \\
\hline 5 & 0.11 & $0.15-0.41$ & 0.01 & $0.13-0.25$ & 0.05 & $0.05-0.11$ & 0.23 & $0.15-0.27$ & 0.72 & $0.3-0.56$ & 1.5 & $0.66-2.25$ \\
\hline 6 & 0.06 & $0.45-1.05$ & 0.08 & $0.17-0.31$ & 0.07 & $0.07-0.27$ & 0.26 & $0.17-0.47$ & 0.72 & $0.27-0.85$ & 1.12 & $1.1-2.22$ \\
\hline 7 & 0.08 & $0.18-0.38$ & 0.06 & $0.18-0.37$ & 0.07 & $0.05-0.11$ & 0.38 & $0.17-0.32$ & 0.74 & $0.46-0.88$ & 1.11 & $1.16-2.13$ \\
\hline 8 & 0.16 & $0.15-0.41$ & 0.05 & $0.13-0.25$ & 0.06 & $0.05-0.11$ & 0.44 & $0.15-0.27$ & 1.7 & $0.3-0.56$ & 1.12 & $0.66-2.25$ \\
\hline 9 & 0.28 & $0.18-0.38$ & 0.03 & $0.18-0.37$ & 0.09 & $0.05-0.11$ & 0.38 & $0.17-0.32$ & 0.66 & $0.46-0.88$ & 1.27 & $1.16-2.13$ \\
\hline 10 & 0.11 & $0.18-0.38$ & 0.08 & $0.18-0.37$ & 0.05 & $0.05-0.11$ & 0.30 & $0.17-0.32$ & 0.57 & $0.46-0.88$ & 1.7 & $1.16-2.13$ \\
\hline 11 & 0.07 & $0.18-0.38$ & 0.03 & $0.18-0.37$ & 0.04 & $0.05-0.11$ & 0.19 & $0.17-0.32$ & 0.52 & $0.46-0.88$ & 0.96 & $1.16-2.13$ \\
\hline
\end{tabular}

The reference ranges for individual patients according to age of patients are displayed in next column. Controls are constituted in the three major groups (0-2 years old; 3-18 years old and adults). Alterations in patient's complexes activities are shown in bold. Abbreviations: $\mathrm{Cl}$ complex I, NADH coenzyme Q reductase; $\mathrm{Cl}+$ III complex I + III, NADH cytochrome c reductase; CII complex II, succinate:coenzyme Q reductase; CII + III complex II + III, succinate:cytochrome C oxidoreductase; CIII complex III, coenzyme Q:cytochrome $c$ oxidoreductase; CIV complex IV, cytochrome $c$ oxidase; CS citrate synthase 
A

C

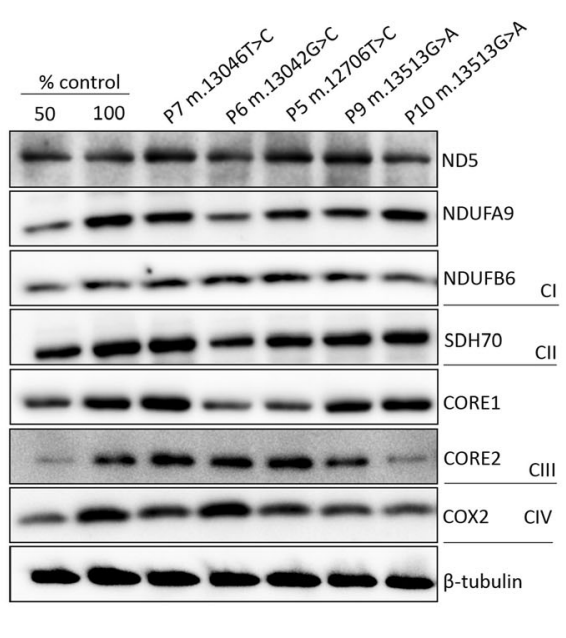

B
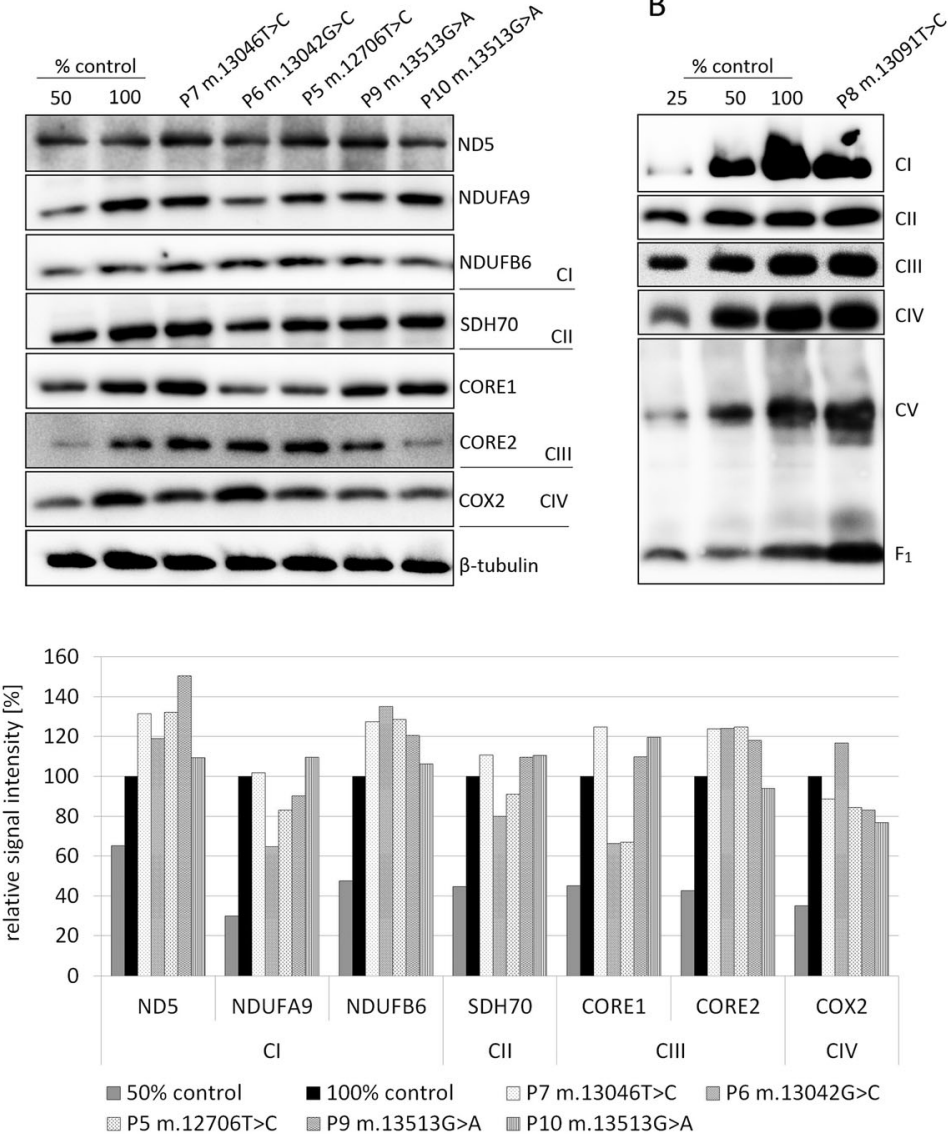

D

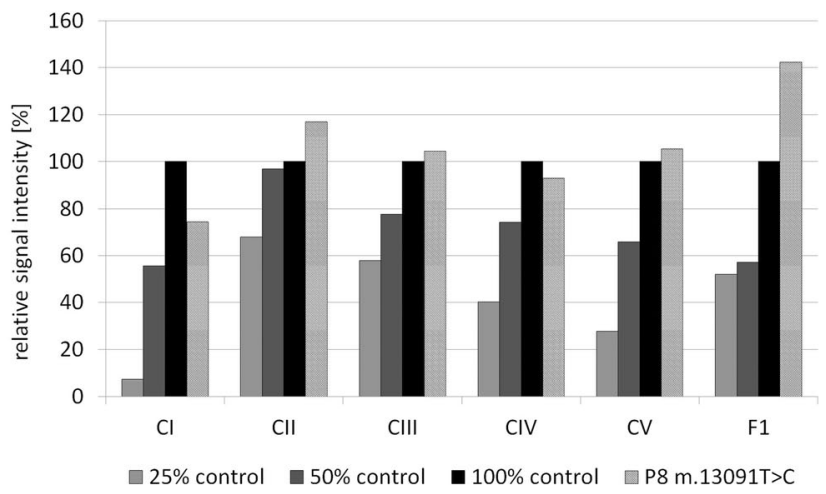

Fig. 2 Protein analysis in six patients with heteroplasmic mutations in MT-ND5 gene. a Comparison of steady-state levels of several OXPHOSrelated proteins in P5, P6, P7, P9 and P10 in fibroblasts using SDS-PAGE/WB. As a control was used primary dermal fibroblasts (ATCC ${ }^{\circledR}$ PCS-201$010^{\mathrm{TM}} \mathrm{M}, 50$ and $100 \%$ demonstrate loading dose of protein amount. b BN-PAGE/WB of OXPHOS complexes in isolated mitochondria from the muscle of P8. As a control was used human muscle mitochondria from heathy adult, 25; 50 and 100\% demonstrate loading dose of protein amount. c The quantification of western blot signals from A by densitometric analysis. Relative signals intensity of individual OXPHOS antibodies were normalized to intensity of loading control $\beta$-tubulin. $\mathbf{d}$ The quantification of western blot signals from B by densitometric analysis

load of m.13091 T > C was present in urine, while blood, buccal smears and hair follicles were negative. The mutational load in samples from the patient's sister was below the detection limit of the method.

\section{Discussion}

Maternally inherited mutations in $M T-N D$, genes for structural subunits of $\mathrm{CI}$, may be found in $>30 \%$ of patients with isolated CI deficiency [16, 17]. Most of them 

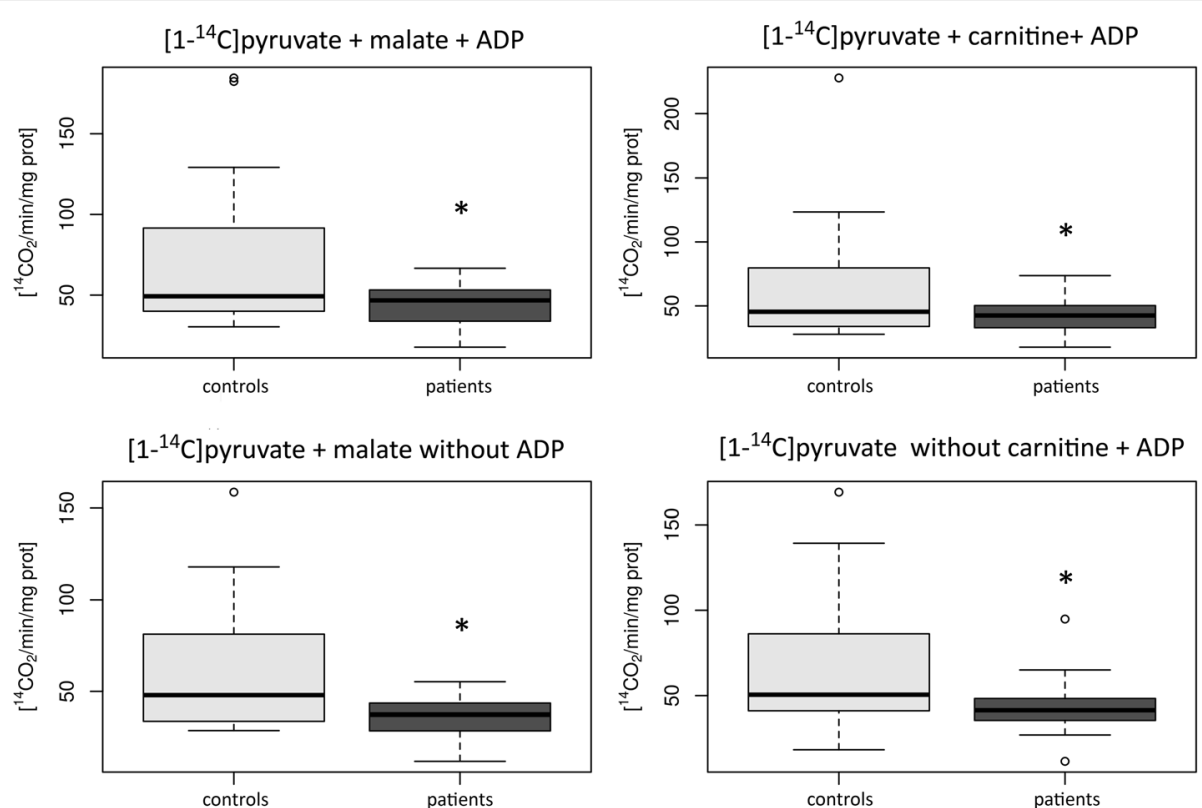

Fig. 3 Oxidation rate of 4 different MEGS incubations containing $\left[1-{ }^{14} \mathrm{C}\right]$ pyruvate in cultivated skin fibroblasts. Patient group consists of 8 patients (P1, P2, P4, P6, P7, and P9-P11) with complex I deficiency and heteroplasmic mutations in MT-ND1, MT-ND3 and MT-ND5 genes. Control group consists of 10 age-related controls. In the group of patients, oxidation rate of all four displayed MEGS incubations are significantly decreased $\left({ }^{*} p<0.05\right)$. Open circles displayed suspected outliers

have LHON syndrome with isolated optic neuropathy due to the prevalent mutations $\mathrm{m} .11777 \mathrm{G}>\mathrm{A}$ in $M T$ ND4, m.3460G $>\mathrm{A}$ in MT-ND1, or m.14484 $\mathrm{T}>\mathrm{C}$ in MT-ND6. Multisystem mitochondrial disorders are less frequent. The MT-ND5 gene appears to be clearly a hotspot for disease-causing mutations [18], and the m.13513G > A mutation is one of the most common [19]. Although the MT-ND5 gene is the largest of the mtDNA-encoded genes for CI (1812 bp), this alone does not explain the increased number of mutations in this gene compared with other mitochondrial genes [20]. Still, it corresponds well with the results of molecular analyses in our families with multisystem MD, because most of them have mutations in the MT-ND5 gene, including one girl with the novel heteroplasmic mutation m.13091 T > C. Similarly to other reports [19, 21, 22] also in our group of patients, the most frequent mutation in the MT-ND5 gene was m.13513G > A. The second most common group of mutations in our subjects were mutations in the MT-ND1 gene, found in three patients. One of the mutations, m.3946G > A, was the first within MT-ND genes associated with the MELAS syndrome phenotype [23].

The phenotype in patients with CI deficiency may have overlapping features of different mitochondrial syndromes. Our patients manifested, most frequently during childhood or adolescence, with LS with progressive motor and subsequent mental deterioration, or with MELAS syndrome, lactic acidosis and attacks of stroke- like episodes. Less frequent was the onset of optic neuropathy followed with multisystem symptoms representing LHON/MELAS overlap syndrome. Hypertrophic CMP with or without WPW syndrome is not rare in patients with the mutation m.13513G > A $[19,21,22,24$, 25]; we observed it in 2 patients. Severe rhabdomyolysis was observed only once, in P7.

Clinical symptoms in P8 with MELAS syndrome and the novel heteroplasmic variant $\mathrm{m} .13091 \mathrm{~T}>\mathrm{C}$ in $M T$ ND5 started with attacks of migraine at the age of 10 years and stroke-like episodes at the age of 26 years. In addition, she developed myopathy, optic neuropathy and secondary epilepsy. This course of the disease is compatible with our observation that any symptom from the broad phenotypic spectrum of MELAS syndrome may come first and stay isolated for a long period of time [26].

Three patients with mutations in MT-ND1 also developed an acute encephalitic episode characterized by an acute qualitative alteration of consciousness with hyporesponsivity and profound hypotonia. This may have been caused by selective neuronal impairment due to energetic deprivation, leading to reversible ischaemic damage that lacks evidence of decreased tissue perfusion [27]. Some patients may recover to a normal or nearly normal condition as before the event $[1,19,22]$. Of some interest is the Wernicke's aphasia in P5, with mutation m.12706 T > C in MT-ND5, which was characterized by a difficulty of understanding written and spoken language. The cause of Wernicke's aphasia is usually an 
ischaemic stroke affecting the Wernicke's area in the posterior temporal lobe of the dominant hemisphere perfused by branches of the middle cerebral artery [28]. MRI of the brain revealed a typical ischemic hyperintensity in the left temporoparietal region. In contrast, MELAS lesions do not follow vascular territories or a border zone and the cerebral angiography fails to demonstrate any steno-occlusive lesions [29]. As far as we know, only one mitochondrial patient with mtDNA mutation m.3243G > A has previously been described with Wernicke's aphasia [30].

Vascular dysfunction seems to play an important role in the pathogenesis of MD. However, the affected areas, as observed via neuroimaging, do not always correspond to classic regional vascular distributions; therefore, they are called "stroke-like" lesions. Additionally, other pathophysiological mechanisms may contribute to or be responsible for the development of stroke-like episodes. One of them is a generalized cytopathy caused by critical energy deprivation in neurons and/or glia. The cytotoxicity may lead to either temporary or permanent lesions depending on the level of energetic failure [31, 32]. It was also hypothesized that the abnormal mitochondria in the endothelium may disturb the blood-brain barrier, resulting in altered ion homeostasis, hyperexcitability and focal epileptic activity [33]. Using MRI and ${ }^{1} \mathrm{H}$ magnetic resonance spectroscopy, different white and grey matter lesions may be found in the central nervous system of patients with CI deficiency. Caudate lesions were more common in patients with mtDNA mutations, as opposed to patients with nuclear mutations [34].

LS is characterized by symmetric involvement of the deep grey matter. The most commonly affected structures are the substantia nigra, putamen, nucleus dentatus and the brainstem; however, the thalamus, cerebellum and grey matter of the spinal cord may also be involved, and lesions in white matter structures were also described [35]. The deep grey matter lesions may result from several factors, including ATP depletion, gliosis, high lactate and excessive reactive oxygen species production [36]. In patients with MELAS syndrome, stroke-like lesions with the involvement of grey matter and subcortical white matter typically exhibit a "shifting spread" (appearance, disappearance and re-appearance). These lesions are characterized by asymmetric signal changes crossing vascular territories and an abnormally prominent lactate peak [37, 38]. Some patients also have small lesions in the deep grey matter (either less symmetrical than in LS or unilateral) and calcifications in basal ganglia.

In our patients with maternally inherited CI deficiency, MRI revealed both the involvement of deep grey matter structures and stroke-like lesions similar to other reports [4]. We also demonstrated the coexistence of LS and stroke-like lesions in 4 of the 13 patients, suggesting that an overlap between the LS and MELAS phenotypes is not rare in patients with maternally inherited CI deficiency. Moderate cortical or periventricular atrophy was present in 4 of 13 patients, but these atrophies have been shown to be non-specific in patients with mitochondrial diseases [34].

Grey matter has been estimated to consume approximately 2.5 -fold more ATP than white matter [39]. It is therefore not surprising that the majority of MDs present with predominant involvement of grey matter that may extend to secondary white matter degeneration [36], similar to what was observed in the subcortical regions in some of our patients. It was hypothesized that LS is typical in patients with very high levels of mtDNA mutation heteroplasmy [36]. However, this was only partly supported by our study, as some of our patients with high levels of heteroplasmy manifested stroke-like phenotypes. In fact, isolated LS and LS/MELAS overlap syndromes were present even in some patients with lower levels of heteroplasmy.

\section{Prognosis}

The prognosis in most patients with multisystem MD and CI deficiency is not good. Similarly to other reports $[1,19,22,40]$, our patients with higher levels of heteroplasmy (expressed as the median of heteroplasmy from all analysed tissues) had poorer prognosis with LS phenotype and an earlier onset of the disease, whereas patients with lower heteroplasmy levels developed milder MELAS or LHON/MELAS phenotypes with a later onset $[19,20,41,42]$. On the other hand, LS was also described in some patients with low levels of heteroplasmy [21, 43, 44]. It was shown in patients with MELAS syndrome, including m.13513G > A mutation, that L-arginine infusion during the acute phase of the stroke-like episode may reduce acute symptoms, and oral supplementation with L-arginine and/or L-citruline may prevent further stroke-like episodes, with arginine acting as a nitric oxide donor, reversing the vasospasm [45]. A recent study at the basic research level has shown that in the induced pluripotent stem cells (iPSCs) with $\mathrm{m} .13513 \mathrm{G}>\mathrm{A}$, it is possible to decrease the level of heteroplasmy in iPSCs using transcription activator-like effector nucleases (TALENs) [46].

\section{Biochemical findings}

The activities of $\mathrm{CI}$ and $\mathrm{CI}+\mathrm{III}$ in isolated muscle mitochondria were decreased in most patients. Results of our study suggest that CI + III activity in muscle biopsy normalized to CS activity (serving as the control enzyme) is a good biochemical indicator for CI deficiency. This may be due to the $\mathrm{CI}$ assay measuring only the redox activity of the enzyme, which takes place within the peripheral 
arm, while mutations in the membrane arm subunits (which includes all seven mtDNA-encoded subunits [47]), may theoretically result in ostensibly normal enzymatic activity [1]. In 4/10 patients, CII + III activity was elevated, probably as a compensatory effect of CI deficiency. A similar effect was described in patients with multiple system atrophy with altered biosynthesis of the electron carrier CoQ10 as a consequence of a mutation in the COQ2 gene [48]. The increase of CI activity that they observed could indicate a compensatory mechanism in response to downstream reduction in CII + III activity in cerebellar and occipital white matter [48]. In comparison to spectrophotometric analyses of muscle biopsies, similar measurements in cultivated fibroblasts for the diagnosis of CI deficiency in most of our patients were less predictive. In addition, no correlation was found between the activities and amounts of $\mathrm{CI}$ and $\mathrm{CI}+$ III in the muscle biopsies or cultivated fibroblasts, and the levels of heteroplasmic mutations in MT-ND1, MTND3 and MT-ND5 genes, similar to several other reports [19-21, 44]. Contrarily, a correlation between the level of heteroplasmy and the level of residual CI activity was described in cybrids derived from patients with mutations m.3481G $>\mathrm{A}$ in MT-ND1, m.10158 $\mathrm{T}>\mathrm{C}$ and m.10191 T > C in $M T-N D 3$ and m.13063G > A in $M T$ ND5 [48, 49]. However, the variable expression may be caused by different nuclear backgrounds, mtDNA haplotypes, environmental factors or ageing [43].

Recently, Kopinski et al. [50] showed how the level of mtDNA heteroplasmy changed the nuclear epigenome through metabolites, in cybrid models with an altered level of m.3243A $>$ G encoded tRNA ${ }^{\text {Leu }}$ in the same nuclear background. They showed changes in nuclear gene expression via histone modification, which is modulated by the level of mitochondrially generated metabolites acetyl-CoA and $\alpha$-ketoglutarate. Levels of those metabolites correlate with histone modifications, which differ across the different levels of heteroplasmy. They also revealed that mtDNA heteroplasmy affects mitochondrial $\mathrm{NAD}^{+} / \mathrm{NADH}$ ratio, which correlates with nuclear histone acetylation. Meanwhile, nuclear $\mathrm{NAD}^{+} / \mathrm{NADH}$ ratio correlates with changes in nDNA and mtDNA transcription. Hence, mutations in mtDNA generate particular metabolites and epigenetic changes at different heteroplasmy levels. This could explain the phenotypic variability of mitochondrial disease [50].

Simultaneous analyses of spectrophotometric and MEGS measurements in muscle biopsies from patients with MDs have already been performed in several studies [15, 51-53]. MEGS analysis is a sensitive method for detection of OXPHOS deficiency and the deficiency of adenine nucleotide translocator or pyruvate dehydrogenase complex (PDHc) in muscle tissue [15, 52]. In our cohort, MEGS analysis revealed disturbances of OXPHOS in all 4 patients where muscle tissue was examined. Moreover, in fibroblasts from 8 patients, MEGS analysis revealed decreased oxidation rates in 4 out of 6 incubations containing $\left[1-{ }^{14} \mathrm{C}\right]$ pyruvate, whereas the oxidation rates of incubations containing $\left[\mathrm{U}-{ }^{14} \mathrm{C}\right]$ malate or $[1$, $4_{-}{ }^{14} \mathrm{C}$ ] succinate remained within the control range. Similarly, diminished $\left[2-{ }^{14} \mathrm{C}\right]$ pyruvate oxidation was also described in 8 out of 11 fibroblast lines from patients with $\mathrm{CI}$ deficiency [54]. The disturbances of $\mathrm{CI}$ led to an increased NADH/NAD ${ }^{+}$ratio, resulting in the inhibition of PDHc activity and thus slowing down of the pyruvate oxidation rate $[55,56]$. Combined evaluation of all MEGS parameters makes it possible to clearly distinguish suspicion for complex I deficiency.

The results of our study suggest that MEGS analyses may serve as a good indicator for CI deficiency and may help to accelerate the diagnostic flow. Both analyses - MEGS and spectrophotometry, especially the activity of complex I + III - are sensitive methods for the recognition of $\mathrm{CI}$ deficiency due to $M T-N D$ mutation in muscle biopsy. In fibroblasts, MEGS seems to be more sensitive. Combining several biochemical methods may improve our understanding of the impact of individual mutations of $M T-N D$ genes on mitochondrial bioenergetics.

\section{Conclusions}

Patients with multisystem MDs due to heteroplasmic mtDNA mutations resulting in isolated CI deficiency represent approximately $11 \%$ of all families with maternally inherited MD diagnosed in our geographical region. MRI of the brain revealed the presence of LS in 6 out of 13 patients (46\%), MELAS syndrome in 3/13 (23\%), and an overlap between both syndromes in 4/13 (31\%). All four patients with mutations in MT-ND1 or $M T-N D 3$ had LS, whereas patients with MT-ND5 mutations presented equally often with LS or MELAS. MEGS, especially oxidation of $\left[1-{ }^{14} \mathrm{C}\right]$ pyruvate might serve as a sensitive indicator of functional impairment due to $M T$ $N D$ mutations in fibroblasts. Early onset of the disease and higher level of mtDNA mutation heteroplasmy were associated with worse prognosis.

\section{Supplementary information}

Supplementary information accompanies this paper at https://doi.org/10 1186/s12887-020-1912-x.

Additional file 1 : Table S1. Structural and Non-Structural Nuclear Genes for Complex I disorders. Table S2. Composition of individual MEGS incubations.

\section{Abbreviations}

BCVA: Best Corrected Visual Acuity; BN-PAGE: Blue Native Polyacrylamide Gel Electrophoresis; Cl + III: Respiratory chain complex I + III (NADH:cytochrome C oxidoreductase); Cl: Respiratory chain complex I (NADH:coenzyme Q oxidoreductase); CII + III: Respiratory chain complex II + II 
(succinate:cytochrome c oxidoreductase); Cll: Respiratory chain complex II (succinate:coenzyme Q oxidoreductase); CIII: Respiratory chain complex III (coenzyme Q:cytochrome c oxidoreductase); CIV: Respiratory chain complex IV (cytochrome c oxidase); CS: Citrate synthase; CSF: Cerebrospinal fluid; HCMP: Hypertrophic cardiomyopathy; iPSCs: Induced Pluripotent Stem Cells; LHON: Leber Hereditary Optic Neuropathy; LS: Leigh syndrome, symmetric necrotic lesions in basal ganglia and/or in the brain stem; MD: Mitochondrial disorders; MEGS: Mitochondrial energy-generating system;

MELAS: Mitochondrial Encephalopathy, Lactic Acidosis and Stroke-like episode; mtDNA: mitochondrial DNA; OXPHOS: Oxidative phosphorylation; PDHc: Pyruvate dehydrogenase complex; RNFL: Retinal nerve fiber layer; SDSPAGE: Sodium dodecyl sulfate polyacrylamide gel electrophoresis; TALENs: Transcription activator-like effector nucleases; WB: Western blot; WPW: Wolf-Parkinson-White syndrome

\section{Acknowledgements}

Special thanks to Daniela Sedlackova and Suzana Knopova for their technical support and to Vaclav Capek for statistical analysis.

\section{Authors' contributions}

TD performed MEGS, SDS-PAGE and BN-PAGE analyses, performed literature search and prepared the manuscript. HK performed the clinical part of the manuscript. JZ interpreted data and prepared the manuscript. $\mathrm{HH}$ performed MEGS in muscle biopsies and supervised the interpretation of OXPHOS functional data. MV and LL collected and interpreted the MRI data, VKV and KB performed mtDNA sequencing and determined heteroplasmy levels of the mutations. TH performed medical examinations of the patients. MT designed the study, interpreted data and revised the manuscript. All authors have read and approved the final manuscript as written and take responsibility for its content.

\section{Funding}

This study was supported by research grants from the Ministry of Health of the Czech Republic (AZV 17-30965A, RVO VFN 64165), and Charles University (Progress Q26/LF1, UNCE 204064, SW260367). The funding bodies were not involved in the design of the study, collection, analysis and interpretation of data, and in the writing of the manuscript.

\section{Availability of data and materials}

The datasets used and/or analysed during the current study are available from the corresponding author on reasonable request.

\section{Ethics approval and consent to participate}

The study was approved by the Ethics Committee of the General University Hospital in Prague and was conducted in agreement with institutional guidelines. Written informed consent for participation in the study was obtained from the patients and parents of affected children.

\section{Consent for publication}

Not applicable.

\section{Competing interests}

The authors declare that they have no competing interests.

\section{Author details}

${ }^{1}$ Department of Pediatrics and Adolescent Medicine, First Faculty of Medicine, Charles University and General University Hospital in Prague, Ke Karlovu 2, 12808 Praha 2, Prague, Czech Republic. ${ }^{2}$ Department of Radiology, First Faculty of Medicine, Charles University and General University Hospital in Prague, Prague, Czech Republic.

\section{Received: 27 August 2019 Accepted: 7 January 2020}

Published online: 29 January 2020

\section{References}

1. Fassone E, Rahman S. Complex I deficiency: clinical features, biochemistry and molecular genetics. J Med Genet. 2012;49(9):578-90.

2. Alston CL, Heidler J, Dibley MG, Kremer LS, Taylor LS, Fratter C, et al. Biallelic mutations in NDUFA6 establish its role in early-onset isolated mitochondrial complex I deficiency. Am J Hum Genet. 2018;103(4):592-601.
3. Kolarova H, Liskova P, Tesarova M, Kucerova Vidrova V, Forgac M, Zamecnik J, et al. Unique presentation of LHON/MELAS overlap syndrome caused by m.13046T>C in MTND5. Ophthalmic Genet. 2016;37(4):419-23.

4. $\mathrm{Ng}$ YS, Lax NZ, Maddison P, Alston CL, Blakely EL, Hepplewhite PD, et al. MT-ND5 mutation exhibits highly variable neurological manifestations at low mutant load. EBioMedicine. 2018;30:86-93.

5. Leng Y, Liu Y, Fang X, Li Y, Yu L, Yuan Y, et al. The mitochondrial DNA $10197 \mathrm{G}$ > a mutation causes MELAS/Leigh overlap syndrome presenting with acute auditory agnosia. Mitochondrial DNA. 2015;26(2):208-12.

6. Vondrackova A, Vesela K, Hansikova H, Zajicova Docekalova D, Rozsypalova E, Zeman J, et al. High-resolution melting analysis of 15 genes in 60 patients with cytochrome-c oxidase deficiency. J Hum Genet. 2012;57(7):442-8.

7. Makinen MW, Lee CP. Biochemical studies of skeletal muscle mitochondria. I. Microanalysis of cytochrome content, oxidative and phosphorylative activities of mammalian skeletal muscle mitochondria. Arch Biochem Biophys. 1968;126(1):75-82

8. Jesina $P$, Tesarová M, Fornůsková $D$, Vojtísková $A$, Pecina $P$, Kaplanová V, et al. Diminished synthesis of subunit a (ATP6) and altered function of ATP synthase and cytochrome c oxidase due to the mtDNA 2 bp microdeletion of TA at positions 9205 and 9206. Biochem J. 2004;383(Pt. 3):561-71.

9. Rustin P, Chretien D, Bourgeron T, Gérard B, Rötig A, Saudubray JM, et al. Biochemical and molecular investigations in respiratory chain deficiencies. Clin Chim Acta. 1994;228(1):35-51.

10. Srere PA. [1] Citrate synthase: [EC 4.1.3.7. Citrate oxaloacetate-lyase (CoA-acetylating)]. In: Lowenstein JM, editor. Methods in Enzymology [Internet]. Academic Press; 1969. p. 3-11. (Citric Acid Cycle; vol. 13). Available from: http://www.sciencedirect.com/science/article/pii/00766 87969130050. [cited 2015 Apr 5]

11. Lowry OH, Rosebrough NJ, Farr AL, Randall RJ. Protein measurement with the Folin phenol reagent. J Biol Chem. 1951;193(1):265-75.

12. Schägger $H$, von Jagow $G$. Blue native electrophoresis for isolation of membrane protein complexes in enzymatically active form. Anal Biochem. 1991;199(2):223-31.

13. Fornuskova D, Brantova O, Tesarova M, Stiburek L, Honzik T, Wenchich L, et al. The impact of mitochondrial tRNA mutations on the amount of ATP synthase differs in the brain compared to other tissues. Biochim Biophys Acta (BBA) - Mol Basis Dis. 2008;1782(5):317-25.

14. Schägger $H$, von Jagow $G$. Tricine-sodium dodecyl sulfate-polyacrylamide gel electrophoresis for the separation of proteins in the range from 1 to 100 kDa. Anal Biochem. 1987;166(2):368-79.

15. Janssen TFJM, Sengers RCA, Wintjes LTM. Ruitenbeek W, Smeitink JAM, et al. measurement of the energy-generating capacity of human muscle mitochondria: diagnostic procedure and application to human pathology. Clin Chem. 2006;52(5):860-71.

16. Swalwell H, Kirby DM, Blakely EL, Mitchell A, Salemi R, Sugiana C, et al Respiratory chain complex I deficiency caused by mitochondrial DNA mutations. Eur J Hum Genet. 2011;19(7):769-75.

17. Ma Y-Y, Li X-Y, Li Z-Q, Song J-Q, Hou J, Li J-H, et al. Clinical, biochemical, and genetic analysis of the mitochondrial respiratory chain complex I deficiency. Medicine (Baltimore). 2018;97(32):e11606.

18. Liolitsa D, Rahman S, Benton S, Carr LJ, Hanna MG. Is the mitochondrial complex I ND5 gene a hot-spot for MELAS causing mutations? Ann Neurol. 2003;53(1):128-32

19. Shanske S, Coku J, Lu J, Ganesh J, Krishna S, Tanji K, et al. The G13513A mutation in the ND5 gene of mitochondrial DNA as a common cause of MELAS or Leigh syndrome: evidence from 12 cases. Arch Neurol. 2008;65(3):368-72.

20. Blok MJ, Spruijt L, de Coo IFM, Schoonderwoerd K, Hendrickx A, Smeets HJ. Mutations in the ND5 subunit of complex I of the mitochondrial DNA are a frequent cause of oxidative phosphorylation disease. J Med Genet. 2007;44(4):e74

21. Sudo A, Honzawa S, Nonaka I, Goto Y. Leigh syndrome caused by mitochondrial DNA G13513A mutation: frequency and clinical features in Japan. J Hum Genet. 2004:49(2):92-6.

22. Chol M. The mitochondrial DNA G13513A MELAS mutation in the NADH dehydrogenase 5 gene is a frequent cause of Leigh-like syndrome with isolated complex I deficiency. J Med Genet. 2003;40(3):188-91.

23. Kirby DM. Mutations of the mitochondrial ND1 gene as a cause of MELAS. Med Genet. 2004;41(10):784-9.

24. Ruiter EM, Siers MH, van den Elzen C, van Engelen BG, Smeitink JAM, Rodenburg RJ, et al. The mitochondrial $13513 \mathrm{G}$ > a mutation is most 
frequent in Leigh syndrome combined with reduced complex I activity, optic atrophy and/or Wolff-Parkinson-white. Eur J Hum Genet EJHG. 2007; 15(2):155-61.

25. Wang S-B, Weng W-C, Lee N-C, Hwu W-L, Fan P-C, Lee W-T. Mutation of mitochondrial DNA G13513A presenting with Leigh syndrome, Wolff-Parkinsonwhite syndrome and cardiomyopathy. Pediatr Neonatol. 2008;49(4):145-9.

26. Dvorakova V, Kolarova H, Magner M, Tesarova M, Hansikova H, Zeman J, et al. The phenotypic spectrum of fifty Czech m.3243A>G carriers. Mol Genet Metab. 2016;118(4):288-95.

27. Tzoulis $C$, Bindoff $L A$. Acute mitochondrial encephalopathy reflects neuronal energy failure irrespective of which genome the genetic defect affects. Brain. 2012;135(12):3627-34.

28. Knepper LE, Biller J, Tranel D, Adams HP, Marsh EE. Etiology of stroke in patients with Wernicke's aphasia. Stroke. 1989;20(12):1730-2.

29. Kim JH, Lim MK, Jeon TY, Rha JH, Rha JH, Eo H, et al. Diffusion and perfusion characteristics of MELAS (mitochondrial myopathy, encephalopathy, lactic acidosis, and stroke-like episode) in thirteen patients. Korean J Radiol. 2011;12(1):15-24.

30. Napolitano A, Salvetti S, Vista M, Lombardi V, Siciliano G, Giraldi C. Long-term treatment with idebenone and riboflavin in a patient with MELAS. Neurol Sci Off J Ital Neurol Soc Ital Soc Clin Neurophysiol. 2000; 21(5 Suppl):S981-2.

31. Ito H, Mori K, Kagami S. Neuroimaging of stroke-like episodes in MELAS. Brain and Development. 2011;33(4):283-8.

32. Sproule DM, Kaufmann P. Mitochondrial encephalopathy, lactic acidosis, and strokelike episodes: basic concepts, clinical phenotype, and therapeutic management of MELAS syndrome. Ann N Y Acad Sci. 2008;1142:133-58,

33. lizuka T, Sakai F, Suzuki N, Hata T, Tsukahara S, Fukuda M, et al. Neuronal hyperexcitability in stroke-like episodes of MELAS syndrome. Neurology. 2002;59(6):816-24.

34. Lebre AS, Rio M, Faivre d'Arcier L, Vernerey D, Landrieu P, Slama A, et al. A common pattern of brain MRI imaging in mitochondrial diseases with complex I deficiency. J Med Genet. 2011;48(1):16-23.

35. Lerman-Sagie T, Leshinsky-Silver E, Watemberg N, Luckman Y, Lev D. White matter involvement in mitochondrial diseases. Mol Genet Metab. 2005;84(2):127-36

36. Lake NJ, Bird MJ, Isohanni P, Paetau A. Leigh syndrome: neuropathology and pathogenesis. J Neuropathol Exp Neurol. 2015;74(6):482-92.

37. Finsterer J, Zarrouk-Mahjoub S. Cerebral imaging in paediatric mitochondrial disorders. Neuroradiol J. 2018;31(6):596-608.

38. Mascalchi M, Montomoli M, Guerrini R. Neuroimaging in mitochondrial disorders. Essays Biochem. 2018:62(3):409-21.

39. Harris JJ, Attwell D. The energetics of CNS white matter. J Neurosci. 2012; 32(1):356-71.

40. Petruzzella V, Di Giacinto G, Scacco S, Piemonte F, Torraco A, Carrozzo R, et al. Atypical Leigh syndrome associated with the D393N mutation in the mitochondrial ND5 subunit. Neurology. 2003;61(7):1017-8.

41. Corona P, Antozzi C, Carrara F, D'Incerti L, Lamantea E, Tiranti V, et al. A novel mtDNA mutation in the ND5 subunit of complex I in two MELAS patients. Ann Neurol. 2001;49(1):106-10.

42. Hanna MG, Nelson IP, Morgan-Hughes JA, Wood NW. MELAS: a new disease associated mitochondrial DNA mutation and evidence for further genetic heterogeneity. J Neurol Neurosurg Psychiatry. 1998;65(4):512-7.

43. Brautbar A, Wang J, Abdenur JE, Chang RC, Thomas JA, Grebe TA, et al. The mitochondrial 13513G>a mutation is associated with Leigh disease phenotypes independent of complex I deficiency in muscle. Mol Genet Metab. 2008;94(4):485-90.

44. Kirby DM, Boneh A, Chow CW, Ohtake A, Ryan MT, Thyagarajan D, et al. Low mutant load of mitochondrial DNA G13513A mutation can cause Leigh's disease. Ann Neurol. 2003;54(4):473-8.

45. Ganetzky RD, Falk MJ. 8-year retrospective analysis of intravenous arginine therapy for acute metabolic strokes in pediatric mitochondrial disease. Mol Genet Metab. 2018;123(3):301-8.

46. Yahata N, Matsumoto Y, Omi M, Yamamoto N, Hata R, et al. Sci Rep. 2017; 7(1):15557.

47. Angerer H, Zwicker K, Wumaier Z, Sokolova L, Heide H, Steger M, et al. A scaffold of accessory subunits links the peripheral arm and the distal proton-pumping module of mitochondrial complex I. Biochem J. 2011; 437(2):279-88
48. Malfatti E, Bugiani M, Invernizzi F, de Souza CF-M, Farina L, Carrara F, et al. Novel mutations of ND genes in complex I deficiency associated with mitochondrial encephalopathy. Brain. 2007;130(7):1894-904.

49. McFarland R, Kirby DM, Fowler KJ, Ohtake A, Ryan MT, Amor DJ, et al. De novo mutations in the mitochondrialND3 gene as a cause of infantile mitochondrial encephalopathy and complex I deficiency. Ann Neurol. 2004; 55(1):58-64.

50. Kopinski PK, Janssen KA, Schaefer PM, Trefely S, Perry CE, Potluri P, et al. Regulation of nuclear epigenome by mitochondrial DNA heteroplasmy. Proc Natl Acad Sci U S A. 2019;116(32):16028-35.

51. Hoefs SJG, Dieteren CEJ, Distelmaier F, Janssen RJRJ, Epplen A, Swarts HGP, et al. NDUFA2 complex I mutation leads to Leigh disease. Am J Hum Genet. 2008;82(6):1306-15.

52. Janssen AJM, Schuelke M, Smeitink JAM, Trijbels FJM, Sengers RCA, Lucke B, et al. Muscle 3243A-->G mutation load and capacity of the mitochondrial energy-generating system. Ann Neurol. 2008;63(4):473-81.

53. Smits $\mathrm{P}$, Antonicka $H$, van Hasselt PM, Weraarpachai W, Haller W, Schreurs $\mathrm{M}$, et al. Mutation in subdomain $\mathrm{G}^{\prime}$ of mitochondrial elongation factor $\mathrm{G} 1$ is associated with combined OXPHOS deficiency in fibroblasts but not in muscle. Eur J Hum Genet. 2011;19(3):275-9.

54. Cameron JM, Levandovskiy V, Mackay N, Robinson BH. Respiratory chain analysis of skin fibroblasts in mitochondrial disease. Mitochondrion. 2004; 4(5-6):387-94.

55. Patel MS, Korotchkina LG. Regulation of the pyruvate dehydrogenase complex. Biochem Soc Trans. 2006;34(Pt 2):217-22.

56. Harris RA, Bowker-Kinley MM, Huang B, Wu P. Regulation of the activity of the pyruvate dehydrogenase complex. Adv Enzym Regul. 2002;42:249-59.

\section{Publisher's Note}

Springer Nature remains neutral with regard to jurisdictional claims in published maps and institutional affiliations.
Ready to submit your research? Choose BMC and benefit from:

- fast, convenient online submission

- thorough peer review by experienced researchers in your field

- rapid publication on acceptance

- support for research data, including large and complex data types

- gold Open Access which fosters wider collaboration and increased citations

- maximum visibility for your research: over $100 \mathrm{M}$ website views per year

At $\mathrm{BMC}$, research is always in progress.

Learn more biomedcentral.com/submissions 